\title{
The effect of bile duct tumor thrombus on the long-term prognosis of hepatocellular carcinoma patients after liver resection: a systematic review and meta-analysis
}

\author{
Jin-Kai Feng ${ }^{1 \#}$, Yu-Xuan Wu ${ }^{2 \#}$, Zhen-Hua Chen ${ }^{3 \#}$, Ju-Xian Sun ${ }^{1}$, Kang Wang ${ }^{1}$, Zong-Tao Chai ${ }^{1}$, Jie Shi ${ }^{1}$, \\ Wei-Xing Guo ${ }^{1}$, Shu-Qun Cheng ${ }^{1}$ \\ ${ }^{1}$ Department of Hepatic Surgery VI, Eastern Hepatobiliary Surgery Hospital, Second Military Medical University, Shanghai, China; ${ }^{2}$ College of \\ Basic Medical Sciences, Second Military Medical University, Shanghai, China; ${ }^{3}$ Department of General Surgery, Zhejiang Provincial Armed Police \\ Corps Hospital, Hangzhou, China \\ Contributions: (I) Conception and design: SQ Cheng, JK Feng, YX Wu, ZH Chen; (II) Administrative support: SQ Cheng; (III) Provision of study \\ materials or patients: JX Sun, K Wang, ZT Chai, J Shi, WX Guo; (IV) Collection and assembly of data: JK Feng, YX Wu; (V) Data analysis and \\ interpretation: JK Feng, YX Wu; (VI) Manuscript writing: All authors; (VII) Final approval of manuscript: All authors. \\ \#These authors contributed equally to this work. \\ Correspondence to: Shu-Qun Cheng, MD, PhD. Eastern Hepatobiliary Surgery Hospital, Second Military Medical University, 225 Changhai Road, \\ Shanghai 200433, China. Email: chengshuqun@aliyun.com.
}

\begin{abstract}
Background: The effect of bile duct tumor thrombus (BDTT) on the postoperative long-term prognosis of hepatocellular carcinoma (HCC) patients is still under debate.

Methods: The PubMed, Embase, Cochrane Library, Web of Science databases were systematically searched to collect the clinicopathologic characteristics, perioperative indices, and postoperative survival outcomes in the BDTT and non-BDTT groups of HCC patients from inception to February 1, 2020. The study outcomes were extracted by two independent investigators.

Results: A total of 15 studies involving 6,484 patients were included. The meta-analysis revealed that the levels of serum total bilirubin and alkaline phosphatase were notably higher in patients with HCC and BDTT than those without BDTT. Meanwhile, HCC patients with BDTT had more aggressive biological characteristics, such as poor tumor differentiation, macrovascular invasion, and lymph node metastasis, as compared to patients without BDTT. The 1-year [odds ratio (OR) 0.39, 95\% confidence interval (CI): 0.310.48, $\mathrm{P}<0.01$ ], 3-year (OR 0.33, 95\% CI: 0.22-0.51, $\mathrm{P}<0.01$ ) and 5-year overall survival (OS) rates (OR 0.31, 95\% CI: $0.20-0.49, \mathrm{P}<0.01$ ) of the BDTT group were significantly worse than those of the non-BDTT group. The hazard ratio of HCC with BDTT was 4.27 (95\% CI: 3.47-5.26, P<0.01) within 5 years after hepatectomy. Conclusions: HCC patients with BDTT had worse OS compared to patients free of BDTT after surgery. BDTT may be a potential prognostic factor for HCC patients.
\end{abstract}

Keywords: Bile duct tumor thrombus (BDTT); hepatocellular carcinoma (HCC); liver resection; overall survival (OS); prognosis

Submitted Jun 4, 2020. Accepted for publication Oct 18, 2020.

doi: $10.21037 / \mathrm{atm}-20-4698$

View this article at: http://dx.doi.org/10.21037/atm-20-4698

\section{Introduction}

Hepatocellular carcinoma (HCC) is the sixth most common malignancy and the fourth leading cause of cancerrelated mortality worldwide (1). HCC has a propensity to invade vasculature systems, which leads to intrahepatic metastasis and extrahepatic spread. A series of studies have demonstrated that macrovascular tumor thrombus is a crucial predisposing factor for HCC patients, and liver 
resection may provide a survival benefit in select $\mathrm{HCC}$ patients with vascular invasion $(2,3)$.

HCC also invades adjacent intra- and extrahepatic biliary tree to form bile duct tumor thrombus (BDTT). BDTT is defined as an intraductal neoplasm, which consists of HCC cells and lining biliary epithelial cells under microscope. BDTT is primarily classified as microscopic and macroscopic tumor thrombus according to its anatomical location in the biliary tract (4). The prevalence of BDTT ranges from $0.5 \%$ to $12.9 \%$ in surgical specimens obtained from HCC (5). The incidence of BDTT in HCC is relatively low, and it is frequently accompanied by concurrent portal vein or hepatic vein invasion. Therefore, our understanding of BDTT is often limited.

To our knowledge, several studies have demonstrated that BDTT is associated with poor prognosis and should be considered an indicator of worse overall survival (OS) following liver resection for HCC patients (6-8). Nevertheless, some high-quality studies have concluded that HCC patients with BDTT can reach similar longterm outcomes after curative-intent surgery (9-11). Consequently, the survival outcomes for HCC patients with BDTT remain controversial.

Herein, we present the first comprehensive systematic review and meta-analysis, in order to compare the clinicopathologic characteristics, perioperative indices and postoperative OS between HCC patients with and without BDTT, and to further explore the prognostic effect of BDTT on HCC, with the aim to provide relevant guidance for clinical practice. The following article is performed on the basis of the Cochrane Collaboration recommendations. We present the following article in accordance with the PRISMA reporting checklist (available at http://dx.doi. org/10.21037/atm-20-4698) (12,13).

\section{Methods}

\section{Study protocol and search strategy}

The PubMed, Embase, Cochrane Library, and Web of Science databases were systematically searched for relevant articles from inception to February 1, 2020. Other potential records were identified using Scopus and Google Scholar. In addition, the reference lists of the retrieved articles were screened carefully and searched manually to identify more relevant studies. Medical Subject Headings (MeSH) terms and free-text terms were used in combination. The following MeSH terms were used: (carcinoma, hepatocellular AND bile duct thrombus). The following free-text terms were used: ((hepatocellular carcinoma OR liver cancer OR hepatic cancer OR liver neoplasm OR liver tumor OR hepatic tumor OR hepatocarcinoma OR liver cell carcinoma OR HCC OR hepatoma) AND (bile duct tumor thromb* OR bile duct tumour thromb* OR biliary tumor thromb* OR biliary tumour thromb* OR bile duct invasion OR biliary invasion)). The retrieved articles were imported to Endnote X9 software for further screening.

\section{Selection criteria}

The inclusion criteria were as follows: (I) clinical studies that compared long-term outcomes between HCC patients with and without BDTT after liver resection; (II) studies with sufficient data available, such as demographics, baseline characteristics, median survival time (MST) and OS outcomes.

The exclusion criteria were as follows: (I) non-HCC; (II) recurrent or secondary HCC; (III) patients underwent adjuvant therapy or liver transplantation; (IV) literature focusing on diagnostic techniques of BDTT; (V) basic experimental studies involving BDTT; (VI) no survival outcomes or curves reported to calculate odds ratio (OR) or hazard ratio (HR); (VII) full-text articles not available; (VIII) case reports, conference abstracts, narrative reviews, comments, letters or other documents unrelated to the topic.

\section{Data extraction}

Two independent investigators (JK Feng, YX Wu) screened the titles and abstracts of all the retrieved citations to identify potentially relevant studies. Full-text articles were then obtained for further review. In cases where disagreement occurred, a third author ( $\mathrm{ZH}$ Chen) was invited to obtain a consensus. A data extraction table was created after repeated discussion and revision. Two authors (JK Feng and YX Wu) extracted the data from original articles into the table. The accuracy and completeness of the data were checked by a third author (ZH Chen).

The contents of the extracted data were composed of the following information: (I) basic information of the enrolled studies, including authors' names, year and country of publication, study design, sample size, gender and age of subjects, and follow-up time; (II) clinicopathologic characteristics, including hepatitis B surface antigen (HBsAg), Child-Pugh classification, the levels of albumin, 
total bilirubin, alkaline phosphatase (ALP) and $\alpha$-fetoprotein (AFP), cirrhosis, tumor size and number, encapsulation, stage and differentiation of the tumor, macrovascular invasion and lymph node metastasis; (III) perioperative indices, including surgical mode (anatomic resection, $\mathrm{R} 0$ resection), operating time, intraoperative blood loss, hospital stay, postoperative morbidity and mortality; (IV) long-term survival outcomes, including the 1-, 3-, and 5 -year OS rates. Particularly, the following characteristics of the BDTT group were extracted: (I) the number of patients with macroscopic or microscopic BDTT; (II) the number of patients with or without obstructive jaundice; (III) the detailed categorizing protocols of BDTT.

\section{Quality assessment}

The Newcastle-Ottawa Scale (NOS) was used to assess the methodologic quality of the included retrospective cohort studies. This scale evaluates studies on the following aspects: (I) selection of the exposed and non-exposed cohorts (4 scores); (II) comparability of different cohorts (2 scores); (III) measurement of outcomes and follow-up (3 scores). If the total score was 7 or greater, the study was of high quality (14). This process was also conducted by two authors (JK Feng, YX Wu). When disagreement in scoring occurred, a third author ( $\mathrm{ZH}$ Chen) participated in the discussion, and a final decision was made collectively.

\section{Statistical analysis}

For binary categorical data, the results are expressed as numbers (percentages), and pooled ORs with corresponding 95\% confidence intervals (CIs) were calculated. For continuous variables, the results are presented as mean \pm standard deviation (SD), and weighted mean differences (WMDs) with 95\% CIs were calculated. When the mean and SD were not available for continuous variables, we used the methods described by Hozo et al. (15) to estimate these values. Cumulative meta-analyses were also performed to assess the stability of OR for OS. Moreover, to evaluate the prognostic effect of BDTT on HCC after hepatectomy, pooled HRs with $95 \%$ CIs were extracted using the software Engauge Digitizer (Version 10.3, (C2014 Mark Mitchell) from Kaplan-Meier curves using the methods reported by Tierney et al. (16).

Chi-square test and $\mathrm{I}^{2}$ statistic were used to quantify the degree of heterogeneity across different studies, and $\mathrm{I}^{2}>50 \%$ indicated significant heterogeneity (17). Effect sizes with significant heterogeneity were pooled using a randomeffects model. Otherwise, a fixed-effects model was applied. Publication bias of HR was assessed using a funnel plot and Egger's test. Sensitivity analysis was further performed to determine the stability of the overall prognostic effect of HR. Statistical significance was set at a $P$ value less than 0.05 (two-tailed).

All of the above meta-analyses were performed using Stata software (Version 12.0, Stata Corp LP, College Station, TX, USA).

\section{Results}

\section{Study selection procedure}

The detailed process of the identification of eligible studies is shown in Figure 1. A total of 291 studies were identified in our initial broad search. After careful reading of the titles and abstracts of these records, 30 articles were selected for eligibility assessment. Following review of the full text, 15 articles were excluded due to the absence of the nonBDTT group for control $(n=8)$, lack of survival outcomes $(n=4)$ and lack of a full-text article $(n=3)$. Eventually, fifteen studies met our inclusion criteria and were included in this analysis (4-8,10,11,18-25).

\section{Basic characteristics and methodologic quality}

The basic characteristics and NOS scores of the included studies are shown in Table 1. In total, 6,484 HCC patients were included, of whom 478 (7.4\%) had concurrent BDTT and 6,006 (92.6\%) did not. The age of the two groups was comparable. Most patients were male, which accounted for $81.2 \%$ and $83.0 \%$ in the BDTT and non-BDTT groups, respectively. The median follow-up time ranged from 24 to 96 months, with the longest follow-up period exceeding 5 years in all studies.

As shown in Table 1, the NOS scores of the included studies ranged from 5 to 9 , with a mean score of 6.9 points. Nine of the fifteen articles were categorized as high-quality studies (4-6,11,19-23). The following listed reasons affected the quality: (I) insufficient description of the methods to identify the exposure factor (18); (II) no control for important confounding factors $(4,7,8,10,18-21,24,25)$; (III) not exhibiting appropriate plans to assess outcomes $(6-8,18,24,25)$; (IV) lack of description of lost to follow-up (4-8,10,18-25).

Table S1 displays the details of the BDTT group and 


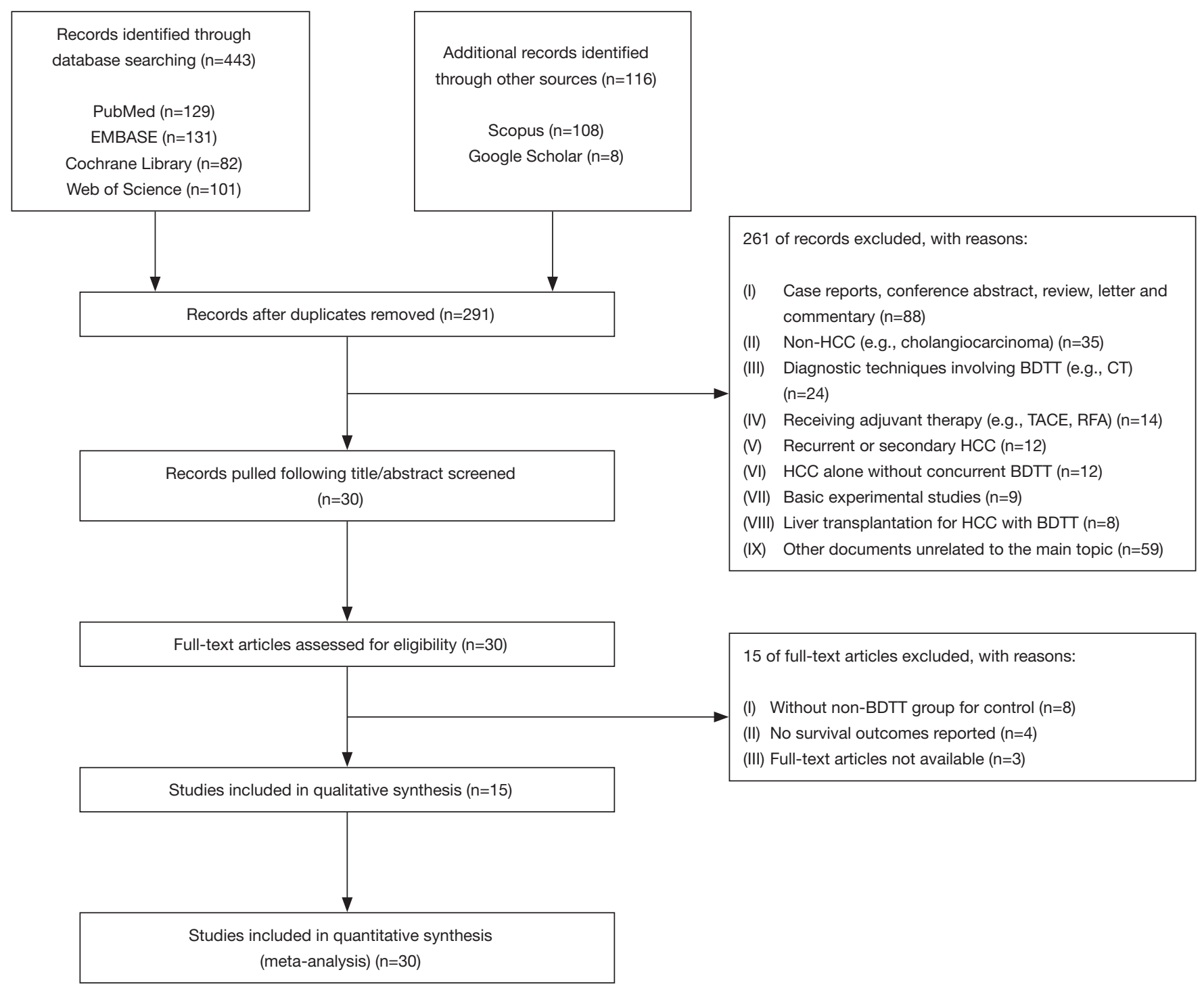

Figure 1 PRISMA flow diagram of the identification process for eligible studies. HCC, hepatocellular carcinoma; BDTT, bile duct tumor thrombus; CT, computed tomography; TACE, transarterial chemoembolization; RFA, radiofrequency ablation.

describes the classification method of BDTT in each of the included studies. As shown in Table S1, four of the fifteen studies contained solely macroscopic BDTT $(4,10,18,21)$, one study only consisted of microscopic BDTT (23), eight studies included both macroscopic and microscopic BDTT (5-8,19,20,24,25), and the remaining studies did not provide the number of patients with macroscopic or microscopic BDTT $(11,22)$. In the BDTT group, $74.8 \%$ (305/408) patients had macroscopic BDTT, and 49.1\% (201/409) patients were complicated with obstructive jaundice on the first admission to hospital.

\section{Clinicopathologic characteristics}

As presented in Table 2, no significant differences were found between the BDTT and non-BDTT groups in regard to HBsAg positivity, AFP level, presence of cirrhosis, number and diameter of primary tumor, or encapsulation. The proportions of Child-Pugh class A (OR 0.50, 95\% CI: 0.26-0.98, $\mathrm{P}=0.04$ ), well or moderate tumor differentiation (OR $0.49,95 \%$ CI: $0.37-0.65, \mathrm{P}<0.01$ ) and the level of albumin (WMD $-1.82,95 \% \mathrm{CI}:-3.31$ to $-0.33, \mathrm{P}=0.02$ ) were significantly lower in the BDTT group; whereas the levels of total bilirubin (WMD 2.18, 95\% CI: 1.56-2.81, 


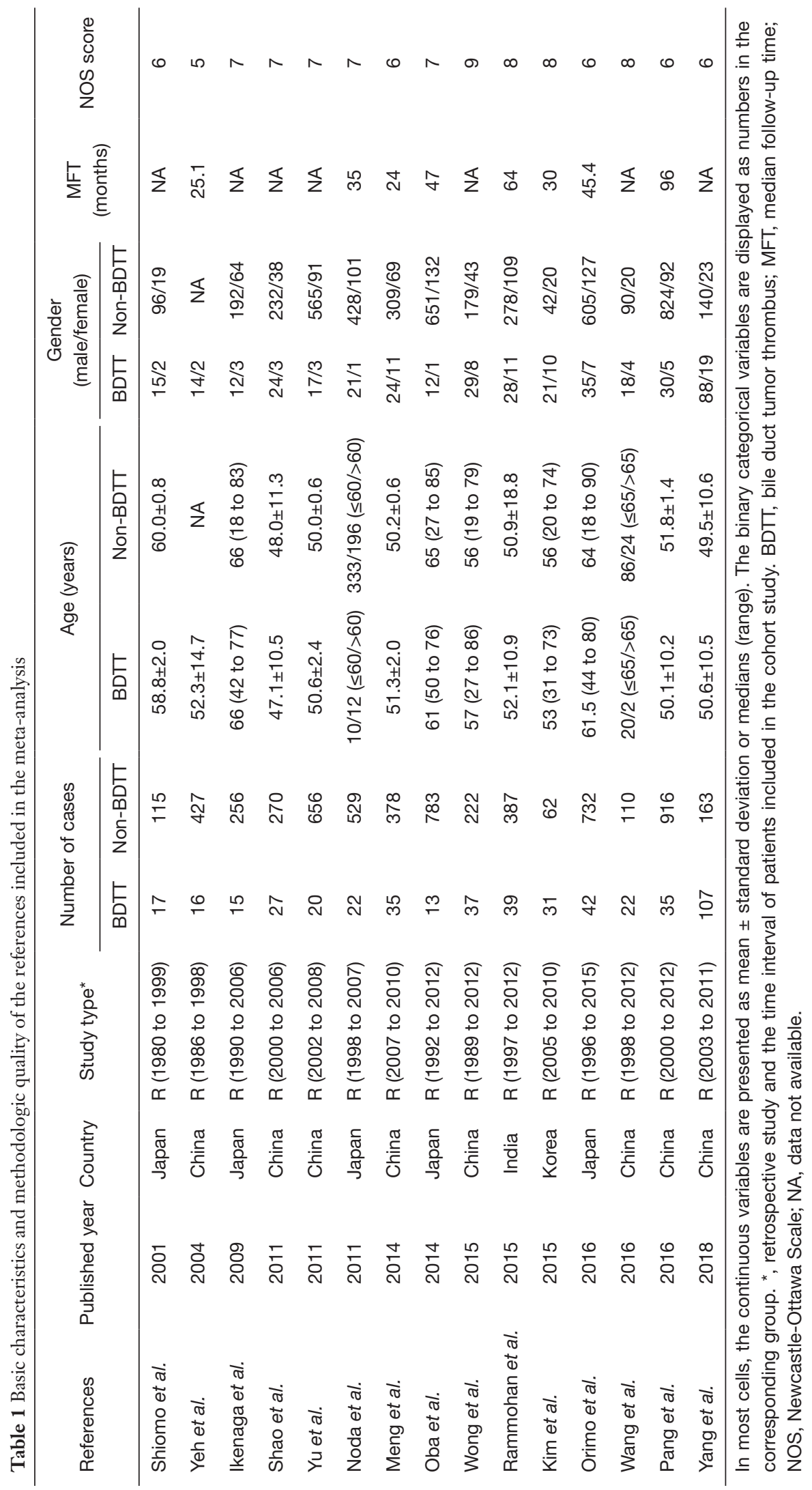


Table 2 Summary of meta-analysis comparing HCC patients with and without BDTT

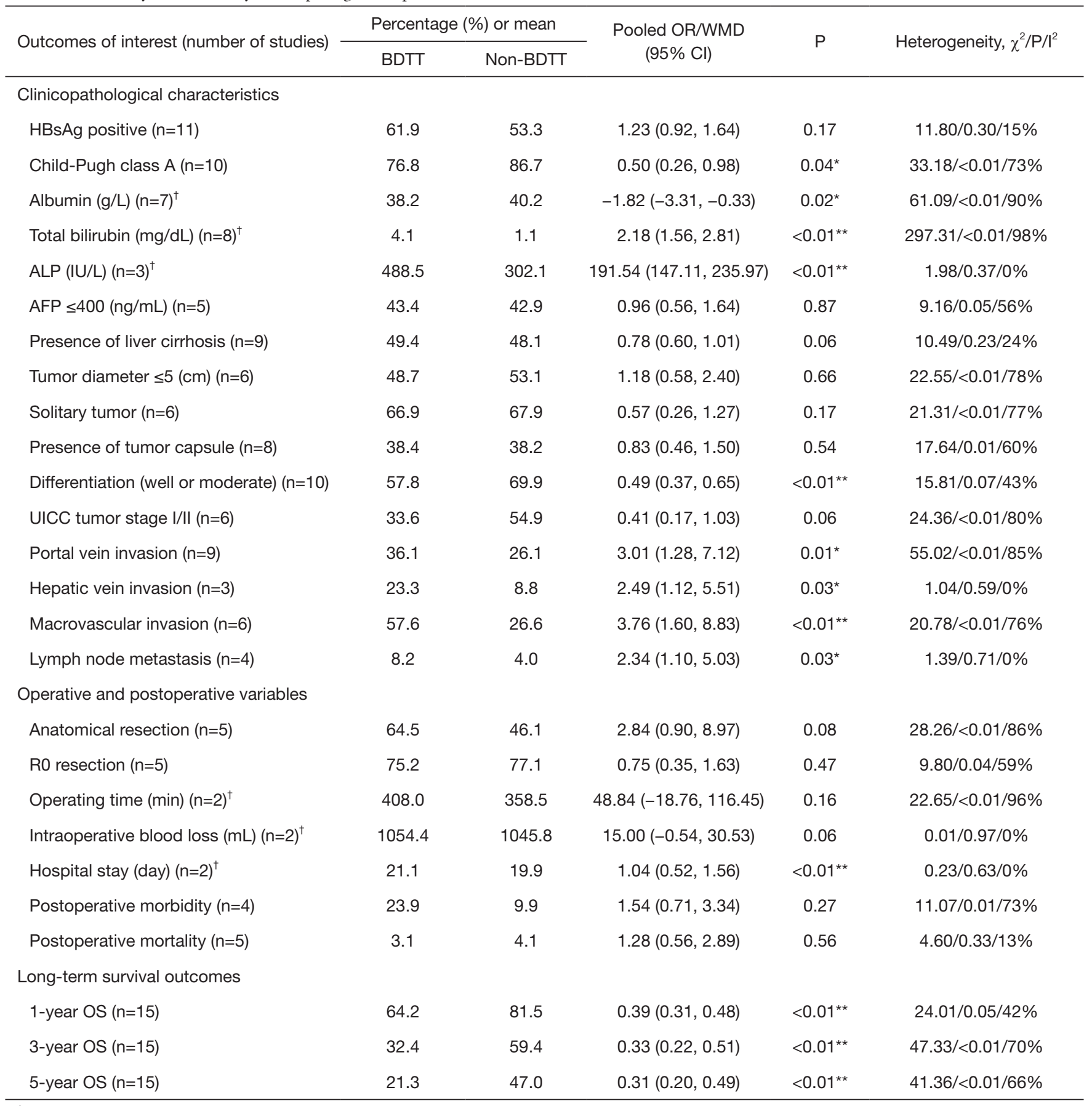

${ }^{\dagger}$, these data were continuous variables and presented as mean; ${ }^{*}, \mathrm{P}<0.05 ;{ }^{* *}, \mathrm{P}<0.01$. HCC, hepatocellular carcinoma; BDTT, bile duct tumor thrombus; OR, odds ratio; WMD, weighted mean difference; HBsAg, hepatitis B surface antigen; ALP, alkaline phosphatase; AFP, $\alpha$-fetoprotein; UICC, Union for International Cancer Control; OS, overall survival. 
$\mathrm{P}<0.01$ ) and ALP (WMD 191.54, 95\% CI: 147.11-235.97, $\mathrm{P}<0.01)$, and the percentages of portal vein invasion (OR 3.01, 95\% CI: $1.28-7.12, \mathrm{P}=0.01)$, hepatic vein invasion (OR 2.49, 95\% CI: $1.12-5.51, \mathrm{P}=0.03)$, macrovascular invasion (OR 3.76, 95\% CI: 1.60-8.83, $\mathrm{P}<0.01$ ) and lymph node metastasis (OR 2.34, 95\% CI: 1.10-5.03, P=0.03) were significantly higher in the BDTT group. The tumor stage of the BDTT group was more advanced compared to the non-BDTT group, but the difference was not statistically significant (OR 0.41, 95\% CI: 0.17-1.03, P=0.06).

\section{Operative and postoperative variables}

There were no significant discrepancies in anatomic resection, R0 hepatectomy, time of surgery, intraoperative blood loss or postoperative morbidity and mortality rates between the two groups. Notably, the duration of hospital stay was markedly longer in the BDTT group versus the non-BDTT group (WMD 1.04, 95\% CI: 0.52-1.56, $\mathrm{P}<0.01$ ) (Table 2).

\section{OS rates}

As shown in Table 2 and Figure 2, the 1-year (64.2\% vs. 81.5\%; OR 0.39, 95\% CI: 0.31-0.48, $\mathrm{P}<0.01$; Figure $2 A$ ), 3 -year (32.4\% vs. 59.4\%; OR 0.30, 95\% CI: 0.22-0.42, $\mathrm{P}<0.01$; Figure $2 B)$ and 5 -year OS rates $(21.3 \%$ vs. $47.0 \%$; OR 0.31 , 95\% CI: $0.20-0.49, \mathrm{P}<0.01$; Figure $2 C$ ) of the BDTT group after liver resection were significantly lower compared to the non-BDTT group. Figure $2 D, E, F$ shows the forest plots of cumulative meta-analyses of ORs for 1-, 3-, and 5-year OS, respectively. The results showed that the significant differences in favor of the non-BDTT group were initially observed in the studies published in 2009, 2004 and 2004, respectively. Then the 95\% CIs developed in a roughly narrowed trend, which suggests that the effect sizes became stable. The detailed survival outcomes of HCC patients with or without BDTT are displayed in Table S2.

\section{Hazard ratio and long-term prognosis}

To further evaluate the impact of BDTT on the longterm prognosis of HCC patients, we calculated HRs within 5 years after surgery for every included study and combined the data using forest plots. As shown in Figure 3, HCC patients with BDTT had worse long-term prognosis after hepatectomy with a pooled HR of 4.27 (95\% CI: 3.47-5.26, $\left.\mathrm{P}<0.01 ; \mathrm{I}^{2}=50.3 \%\right)$, which suggests that BDTT may be a potential risk factor for HCC.

\section{Publication bias and sensitivity analysis for $H R$}

As shown in Figure S1, a visual inspection of the funnel plot suggests a symmetric distribution (Figure S1A). Egger's test confirmed that there was no significant publication bias $(\mathrm{P}=0.955$; Figure $\mathrm{S} 1 \mathrm{~B})$. Sensitivity analysis (Figure S2) was performed to determine the stability of the overall prognostic effect. The result revealed one study that potentially influenced the pooled HR (11). After exclusion of this study, the heterogeneity between studies was significantly reduced $\left(\mathrm{I}^{2}=3.6 \%, \mathrm{P}=0.41\right)$. The pooled $\mathrm{HR}$ of 4.58 (95\% CI: 3.96-5.28, $\mathrm{P}<0.01$ ) became more positive compared to the initial value.

\section{Discussion}

The present study is the first systematic review and metaanalysis to comprehensively compare the clinicopathologic characteristics, perioperative indices, and survival outcomes between HCC patients with and without BDTT. We first reported the pooled $\mathrm{HR}$ of $\mathrm{HCC}$ patients with BDTT within 5 years following hepatectomy. In addition, we reviewed 15 well-designed studies of 6,484 HCC patients, including 478 cases with HCC and BDTT, which is the largest sample size to date on this subject.

In the current study, we found that patients with HCC and BDTT had worse liver function, lower levels of albumin, higher levels of total bilirubin and ALP, and a longer duration of hospital stay. HCC with BDTT was associated with more aggressive biological characteristics, such as advanced tumor stage, poor differentiation, macrovascular invasion, and lymph node metastasis, all of which are recognized critical factors that restrict longterm survival. These results are consistent with previous findings $(21,26)$.

HCC patients with BDTT are frequently complicated with obstructive jaundice at first presentation, and the incidence of this combination ranged from $27 \%$ to $70 \%$ in previous studies $(6,20,21,27,28)$. Generally, when BDTT extends beyond the confluence of the left and right hepatic ducts, the symptoms of obstructive jaundice will appear in patients with HCC and BDTT. The cause of jaundice resulting from BDTT is primarily a tumor thrombus that continuously extends to the extrahepatic bile duct, a dislodged BDTT blocking the common bile duct, or blood clots formed by haemobilia occluding the outflow of biliary 
A

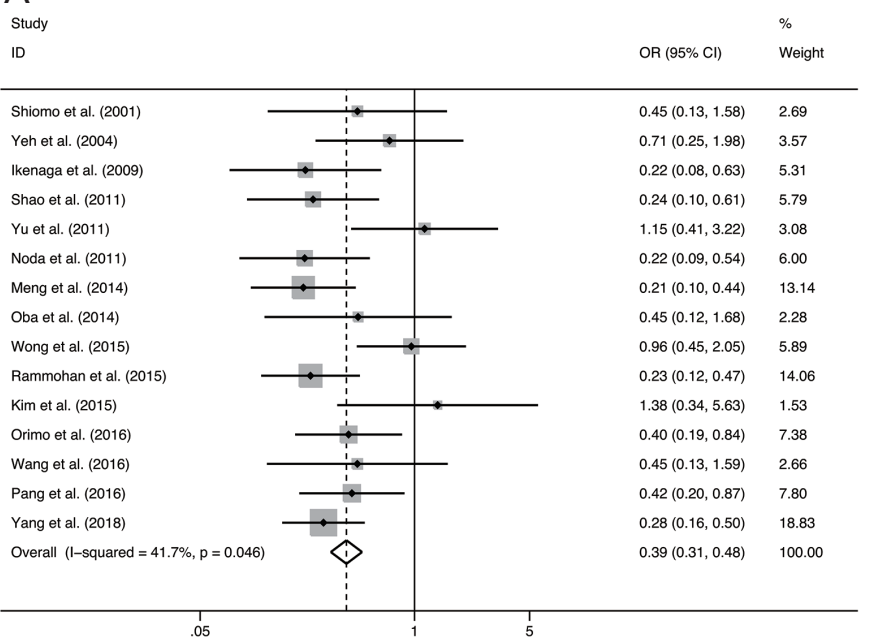

C

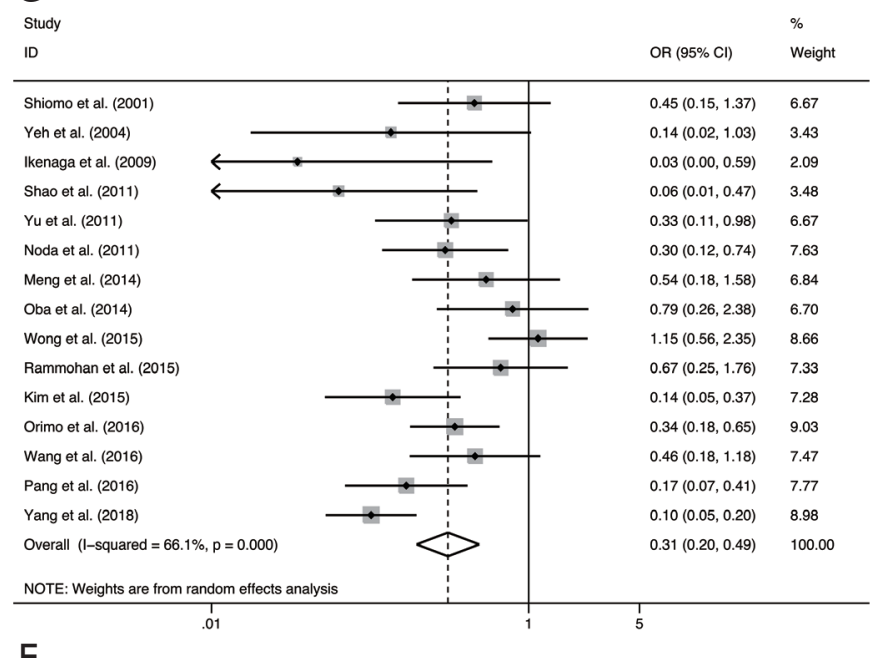

E

Study

ID

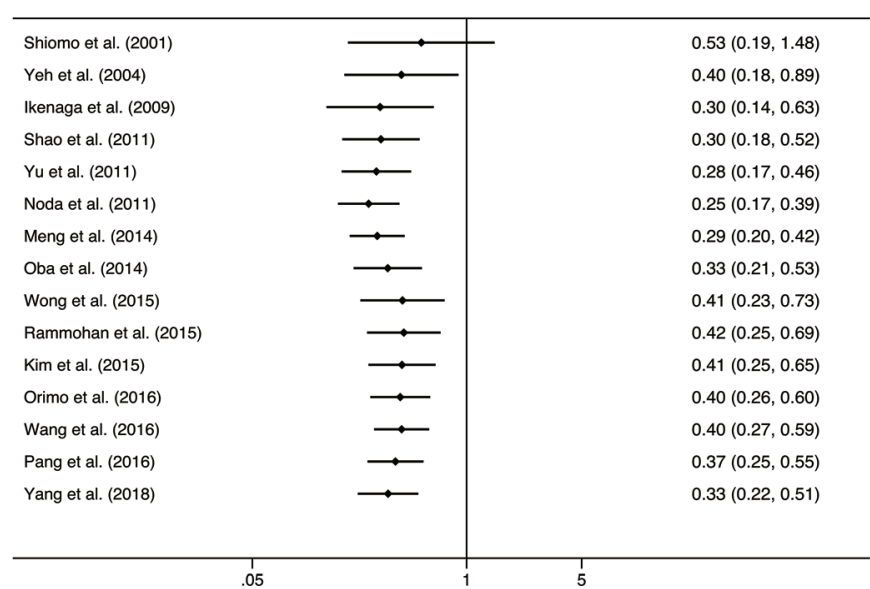

B

Study $\%$

ID $\quad$ OR $(95 \% \mathrm{Cl}) \quad$ Weight

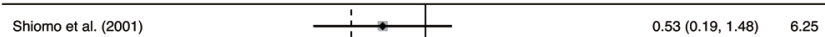

\begin{tabular}{lllll} 
Yeh et al. (2004) & $0.26(0.07,0.93)$ & 5.22 \\
\hdashline & $0.15(0.04,0.54)$ & 5.14
\end{tabular}

Ikenaga et al. (2009) $\longleftarrow:-150.15(0.04,0.54) \quad 5.14$

Shao et al. (2011) $\quad \begin{array}{llll} & 0.30(0.12,0.73) & 6.86\end{array}$

\begin{tabular}{llll} 
Shao et al. (2011) & $0.30(0.12,0.73)$ & 6.86 \\
Yu et al. (2011) & $0.22(0.07,0.66)$ & 5.89 \\
\hline & & $0.17(0.07,0.43)$ & 6.74
\end{tabular}

Noda et al. (2011) $\longrightarrow+\begin{array}{rr}0.17(0.07,0.43) & 6.74 \\ \longrightarrow & 1\end{array}$

Meng et al. (2014) $0.46(0.20,1.08) \quad 7.04$

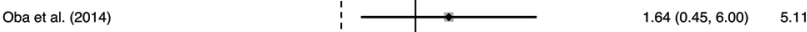

Wong et al. (2015) $\quad \begin{array}{rrrr}-1.44(0.71,2.89) & 7.78\end{array}$

$\begin{array}{lllll}\text { Rang et al. (2015) } & & 1.44(0.71,2.89) & 7.78 \\ \text { Rammohan et al. (2015) } & 0.46(0.21,1.03) & 7.28\end{array}$

Kim et al. (2015)

Orimo et al. (2016) $\quad \begin{array}{lll}0.31(0.17,0.59) & 8.11\end{array}$

Wang et al. (2016) $\quad 0.49(0.19,1.23) \quad 6.70$

Pang et al. (2016) $\quad 0.14(0.06,0.33) \quad 7.11$

\begin{tabular}{ll|rr} 
& \\
Yang et al. (2018) & $0.11(0.06,0.20)$ & 8.35
\end{tabular}

Overall (I-squared $=70.4 \%, p=0.000) \quad D \quad 0.33(0.22,0.51) \quad 100.00$

\begin{tabular}{c:c|c|c|}
\hline OTE: Weights are from random effects analysis & & \multicolumn{2}{|c|}{} \\
\hline 1 & & 1 & 5
\end{tabular}

D

Study

\begin{tabular}{|c|c|c|}
\hline ID & & OR $(95 \% \mathrm{Cl})$ \\
\hline Shiomo et al. (2001) & & $0.45(0.13,1.58)$ \\
\hline Yeh et al. (2004) & & $0.60(0.27,1.32)$ \\
\hline Ikenaga et al. (2009) & $\longrightarrow$ & $0.42(0.23,0.79)$ \\
\hline Shao et al. (2011) & $\longrightarrow$ & $0.36(0.22,0.61)$ \\
\hline Yu et al. (2011) & $\longrightarrow$ & $0.48(0.31,0.76)$ \\
\hline Noda et al. (2011) & $\longrightarrow$ & $0.42(0.28,0.63)$ \\
\hline Meng et al. (2014) & $\longrightarrow$ & $0.35(0.25,0.50)$ \\
\hline Oba et al. (2014) & $\longrightarrow$ & $0.36(0.25,0.50)$ \\
\hline Wong et al. (2015) & 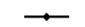 & $0.43(0.32,0.59)$ \\
\hline Rammohan et al. (2015) & $\rightarrow$ & $0.39(0.29,0.51)$ \\
\hline Kim et al. (2015) & $\rightarrow$ & $0.41(0.31,0.54)$ \\
\hline Orimo et al. (2016) & $\rightarrow$ & $0.41(0.32,0.53)$ \\
\hline Wang et al. (2016) & $\rightarrow$ & $0.41(0.32,0.53)$ \\
\hline Pang et al. (2016) & $\rightarrow$ & $0.41(0.32,0.52)$ \\
\hline Yang et al. (2018) & $\rightarrow$ & $0.39(0.31,0.48)$ \\
\hline
\end{tabular}

$\mathrm{F}$

Study

OR $(95 \% \mathrm{Cl})$

\begin{tabular}{|c|c|c|}
\hline Shiomo et al. (2001) & $\longrightarrow$ & $0.45(0.15,1.37)$ \\
\hline Yeh et al. (2004) & & $0.33(0.11,0.98)$ \\
\hline Ikenaga et al. (2009) & & $0.19(0.04,0.85)$ \\
\hline Shao et al. (2011) & & $0.15(0.04,0.54)$ \\
\hline Yu et al. (2011) & . & $0.21(0.08,0.50)$ \\
\hline Noda et al. (2011) & $\longrightarrow$ & $0.25(0.13,0.47)$ \\
\hline Meng et al. (2014) & $\longrightarrow$ & $0.29(0.16,0.51)$ \\
\hline Oba et al. (2014) & $\longrightarrow$ & $0.33(0.19,0.58)$ \\
\hline Wong et al. (2015) & $\longrightarrow$ & $0.39(0.21,0.71)$ \\
\hline Rammohan et al. (2015) & $\longrightarrow$ & $0.42(0.25,0.73)$ \\
\hline Kim et al. (2015) & $\longrightarrow$ & $0.37(0.21,0.63)$ \\
\hline Orimo et al. (2016) & $\rightarrow$ & $0.37(0.23,0.59)$ \\
\hline Wang et al. (2016) & $\rightarrow$ & $0.38(0.25,0.59)$ \\
\hline Pang et al. (2016) & $\rightarrow$ & $0.36(0.23,0.54)$ \\
\hline Yang et al. (2018) & $\rightarrow$ & $0.31(0.20,0.49)$ \\
\hline
\end{tabular}

Figure 2 Forest plots of the meta-analysis and cumulative meta-analysis for OS. (A) Forest plot of OR of 1-year OS; (B) forest plot of OR of 3-year OS; (C) forest plot of OR of 5-year OS; (D) cumulative meta-analysis of 1-year OS; (E) cumulative meta-analysis of 3-year OS; (F) cumulative meta-analysis of 5 -year OS. OS, overall survival. 


\begin{tabular}{l} 
Study \\
ID \\
\hline Shiomo et al. (2001) \\
Yeh et al. (2004) \\
Ikenaga et al. (2009) \\
Shao et al. (2011) \\
Yu et al. (2011) \\
Noda et al. (2011) \\
Meng et al. (2014) \\
Oba et al. (2014)
\end{tabular}

Figure 3 Forest plot of the hazard ratio for OS of HCC patients with BDTT within 5 years after surgery. BDTT, bile duct tumor thrombus; HCC, hepatocellular carcinoma; OS, overall survival.

tract (29-31). Therefore, it is understandable that the levels of total bilirubin and ALP, as indicators of cholestasis, are significantly higher in this subset of patients. As a result, most HCC patients with BDTT undergo preoperative biliary decompression to alleviate jaundice and improve hepatic function $(32,33)$.

Some recent experimental studies have revealed that genetic silencing of the microRNA-200 family, which are molecular regulators inversely associated with epithelialto-mesenchymal transition (EMT), and overexpression of CCL20, which is a chemokine positively related to the invasion and metastasis of HCC, are much more frequently observed in the samples obtained from HCC patients with BDTT than patients without BDTT $(34,35)$. Besides, since the portal vein, common bile duct and their branches are enclosed together within the Glisson's sheath, tumor cells can invade both structures to form portal vein tumor thrombus (PVTT) and BDTT. All of these factors may partially explain the aggressive biological characteristics that were more commonly encountered in the BDTT group in our study.

As to the outcomes of various treatment modalities, An et al. (36) reported that the 1-, 3-, and 5-year OS rates of HCC patients with BDTT undergoing TACE were $20.4 \%, 6.7 \%$ and $1.3 \%$, respectively. Oba et al. (4) reported that the 1-, 3-, and 5-year OS rates of the BDTT group receiving non-surgical treatments were $14 \%, 5 \%$ and $0 \%$, respectively. The current study found that the pooled OS rates of patients with HCC and BDTT within 1 year, 3 years and 5 years after surgery were $64.2 \%, 32.3 \%$ and $21.3 \%$, respectively. Therefore, the use of aggressive surgical resection in select HCC patients with BDTT appears reasonable to prolong survival.

However, whether HCC patients with BDTT would reach similar long-term survival after surgical treatment compared to patients without BDTT remains controversial. Oba et al. (4) and Wong et al. (11) demonstrated that the two groups had comparable 1-, 3-, and 5-year OS rates following hepatectomy, but other researchers concluded that the postoperative OS of HCC patients with BDTT was significantly shorter than patients without BDTT $(8,21,25)$. Our study provided evidence that HCC with BDTT was associated with significantly inferior 1-, 3-, and 5-year OS.

According to earlier studies, the main obstacle to achieving long-term survival in patients with HCC and BDTT is the high incidence of recurrence after surgery. 
Yang et al. (8) reported that the median recurrence-free survival (RFS) time and cumulative 1-, 3- and 5-year RFS rates in the BDTT group were significantly worse compared to the non-BDTT group. Qin et al. (27) and Zeng et al. (37) reported that $50 \%$ and $43 \%$ of HCC patients with BDTT, respectively, suffered tumor recurrence within the first year after hepatectomy. Consequently, adjuvant or neoadjuvant therapy may be administered to these patients to reduce recurrence rates and improve survival outcomes (38).

Vascular invasion is a well-established risk factor for HCC $(3,39,40)$. However, there is no consensus regarding the prognostic effect of BDTT on the long-term survival of HCC. Wong et al. (11) demonstrated that the presence of BDTT did not influence the prognosis of HCC patients using multivariate analyses. In contrast, Yang et al. (8) noted that macroscopic BDTT was significantly associated with a poor prognosis in HCC patients who underwent liver resection. Our study demonstrated that BDTT was an important predictive factor of a poor prognosis in $\mathrm{HCC}$ patients (HR 4.27, 95\% CI: 3.47-5.26, $\mathrm{P}<0.01 ; \mathrm{I}^{2}=50.3 \%$ ). However, this result should be further verified because of the many confounding factors existing in the analysis.

Several limitations in the present analysis should be considered. First, all of the studies included in this review were retrospective trials with inherent publication and selection bias. Second, all of the research was performed in Asian countries. Therefore, the applicability of these results must be further validated in Western countries. Third, due to different sample sizes and matched methods across studies, some results of this meta-analysis had relatively great heterogeneity. Lastly, this review did not distinguish different types of BDTT, which may influence the final conclusion.

\section{Conclusions}

In conclusion, this systematic review and meta-analysis suggested that the survival outcomes were significantly worse in patients with HCC and BDTT after liver resection than in patients without BDTT. BDTT may be a prognostic factor for HCC patients. Notably, these results must be further validated in more large-scale, well-designed clinical trials.

\section{Acknowledgments}

The authors would like to express their gratitude to American fournal Experts (AFE) (http://www.aje.cn/) for the expert linguistic services provided.

Funding: This study was supported by the Key Project of Natural Science Foundation of China (No. 81730097); the Grants of the Science Fund for Creative Research Groups (No. 81521091); the National Natural Science Foundation of China (No. 81602523).

\section{Footnote}

Reporting Checklist: The authors have completed the PRISMA reporting checklist. Available at http://dx.doi. org/10.21037/atm-20-4698

Peer Review File: Available at http://dx.doi.org/10.21037/ atm-20-4698

Conflicts of Interest: All authors have completed the ICMJE uniform disclosure form (available at http://dx.doi. org/10.21037/atm-20-4698). The authors have no conflicts of interest to declare.

Ethical Statement: The authors are accountable for all aspects of the work in ensuring that the questions related to the accuracy or integrity of any part of the work are appropriately investigated and resolved.

Open Access Statement: This is an Open Access article distributed in accordance with the Creative Commons Attribution-NonCommercial-NoDerivs 4.0 International License (CC BY-NC-ND 4.0), which permits the noncommercial replication and distribution of the article with the strict proviso that no changes or edits are made and the original work is properly cited (including links to both the formal publication through the relevant DOI and the license). See: https://creativecommons.org/licenses/by-nc-nd/4.0/.

\section{References}

1. Bray F, Ferlay J, Soerjomataram I, et al. Global cancer statistics 2018: GLOBOCAN estimates of incidence and mortality worldwide for 36 cancers in 185 countries. CA Cancer J Clin 2018;68:394-424.

2. Kokudo T, Hasegawa K, Matsuyama Y, et al. Survival benefit of liver resection for hepatocellular carcinoma associated with portal vein invasion. J Hepatol 2016;65:938-43.

3. Kokudo T, Hasegawa K, Yamamoto S, et al. Surgical treatment of hepatocellular carcinoma associated with 
hepatic vein tumor thrombosis. J Hepatol 2014;61:583-8.

4. Oba A, Takahashi S, Kato Y, et al. Usefulness of resection for hepatocellular carcinoma with macroscopic bile duct tumor thrombus. Anticancer Res 2014;34:4367-72.

5. Wang DD, Wu LQ, Wang ZS. Prognosis of hepatocellular carcinoma with bile duct tumor thrombus after R0 resection: a matched study. Hepatobiliary Pancreat Dis Int 2016;15:626-32.

6. Ikenaga N, Chijiiwa K, Otani K, et al. Clinicopathologic characteristics of hepatocellular carcinoma with bile duct invasion. J Gastrointest Surg 2009;13:492-7.

7. Meng KW, Dong M, Zhang WG, et al. Clinical characteristics and surgical prognosis of hepatocellular carcinoma with bile duct invasion. Gastroenterol Res Pract 2014;2014:604971.

8. Yang X, Qiu Z, Ran R, et al. Prognostic importance of bile duct invasion in surgical resection with curative intent for hepatocellular carcinoma using PSM analysis. Oncol Lett 2018;16:3593-602.

9. Satoh S, Ikai I, Honda G, et al. Clinicopathologic evaluation of hepatocellular carcinoma with bile duct thrombi. Surgery 2000;128:779-83.

10. Shiomi M, Kamiya J, Nagino M, et al. Hepatocellular carcinoma with biliary tumor thrombi: aggressive operative approach after appropriate preoperative management. Surgery 2001;129:692-8.

11. Wong TCL, Cheung TT, Chok KSH, et al. Outcomes of hepatectomy for hepatocellular carcinoma with bile duct tumour thrombus. HPB (Oxford) 2015;17:401-8.

12. Higgins JP, Altman DG, Gøtzsche PC, et al. The Cochrane Collaboration's tool for assessing risk of bias in randomised trials. BMJ 2011;343:d5928.

13. Moher D, Liberati A, Tetzlaff J, et al. Preferred reporting items for systematic reviews and meta-analyses: the PRISMA statement. BMJ 2009;339:b2535.

14. Yuhara H, Steinmaus C, Cohen S, et al. Is diabetes mellitus an independent risk factor for colon cancer and rectal cancer? Am J Gastroenterol 2011;106:1911-21; quiz 22.

15. Hozo SP, Djulbegovic B, Hozo I. Estimating the mean and variance from the median, range, and the size of a sample. BMC Med Res Methodol 2005;5:13.

16. Tierney JF, Stewart LA, Ghersi D, et al. Practical methods for incorporating summary time-to-event data into metaanalysis. Trials 2007;8:16.

17. Higgins JP, Thompson SG, Deeks JJ, et al. Measuring inconsistency in meta-analyses. BMJ 2003;327:557-60.

18. Yeh CN, Jan YY, Lee WC, et al. Hepatic resection for hepatocellular carcinoma with obstructive jaundice due to biliary tumor thrombi. World J Surg 2004;28:471-5.

19. Shao W, Sui C, Liu Z, et al. Surgical outcome of hepatocellular carcinoma patients with biliary tumor thrombi. World J Surg Oncol 2011;9:2.

20. Yu X, Xu L, Liu C, et al. Clinicopathological characteristics of 20 cases of hepatocellular carcinoma with bile duct tumor thrombi. Dig Dis Sci2011;56:252-9.

21. Noda T, Nagano H, Tomimaru Y, et al. Prognosis of hepatocellular carcinoma with biliary tumor thrombi after liver surgery. Surgery 2011;149:371-7.

22. Rammohan A, Sathyanesan J, Rajendran K, et al. Bile duct thrombi in hepatocellular carcinoma: is aggressive surgery worthwhile? HPB (Oxford) 2015;17:508-13.

23. Kim JM, Kwon CHD, Joh JW, et al. Incidental microscopic bile duct tumor thrombi in hepatocellular carcinoma after curative hepatectomy: a matched study. Medicine 2015;94:e450.

24. Orimo T, Kamiyama T, Yokoo H, et al. Hepatectomy for Hepatocellular Carcinoma with Bile Duct Tumor Thrombus, Including Cases with Obstructive Jaundice. Ann Surg Oncol 2016;23:2627-34.

25. Pang YB, Zhong JH, Luo XL, et al. Clinicopathological characteristics and liver stem cell marker expression in hepatocellular carcinoma involving bile duct tumor thrombi. Tumour Biol 2016;37:5879-84.

26. Navadgi S, Chang C, Bartlett A, et al. Systematic review and meta-analysis of outcomes after liver resection in patients with hepatocellular carcinoma (HCC) with and without bile duct thrombus. HPB (Oxford) 2016;18:312-6.

27. Qin LX, Ma ZC, Wu ZQ, et al. Diagnosis and surgical treatments of hepatocellular carcinoma with tumor thrombosis in bile duct: experience of 34 patients. World J Gastroenterol 2004;10:1397-401.

28. Kim DS, Kim BW, Hatano E, et al. Surgical Outcomes of Hepatocellular Carcinoma With Bile Duct Tumor Thrombus: A Korea-Japan Multicenter Study. Ann Surg 2020;271:913-21.

29. Chen MF, Jan YY, Jeng LB, et al. Obstructive jaundice secondary to ruptured hepatocellular carcinoma into the common bile duct. Surgical experiences of 20 cases. Cancer 1994;73:1335-40.

30. Tantawi B, Cherqui D, Tran van Nhieu J, et al. Surgery for biliary obstruction by tumour thrombus in primary liver cancer. Br J Surg 1996;83:1522-5.

31. Lau W, Leung $\mathrm{K}$, Leung $\mathrm{T}$, et al. A logical approach to hepatocellular carcinoma presenting with jaundice. Ann Surg 1997;225:281-5. 
32. Kubota Y, Seki T, Kunieda K, et al. Biliary endoprosthesis in bile duct obstruction secondary to hepatocellular carcinoma. Abdom Imaging 1993;18:70-5.

33. Lu Z, Sun W, Wen F, et al. Clinical application of percutaneous drainage in treating hepatocellular carcinoma with bile duct tumor thrombus. Contemp Oncol (Pozn) 2013;17:176-83.

34. Yeh TS, Wang F, Chen TC, et al. Expression profile of microRNA-200 family in hepatocellular carcinoma with bile duct tumor thrombus. Ann Surg 2014;259:346-54.

35. Yang X, Ran R, Du J, et al. CCL20 is overexpressed in hepatocellular carcinoma with bile duct tumor thrombus and correlates negatively with surgical outcome. Int J Clin Exp Pathol 2018;11:3977-83.

36. An J, Lee K, Kim K, et al. Clinical features and outcomes of patients with hepatocellular carcinoma complicated with bile duct invasion. Clin Mol Hepatol 2017;23:160-9.

37. Zeng H, Xu L, Wen J, et al. Hepatocellular carcinoma

Cite this article as: Feng $\mathrm{JK}, \mathrm{Wu} Y X$, Chen ZH, Sun JX, Wang K, Chai ZT, Shi J, Guo WX, Cheng SQ. The effect of bile duct tumor thrombus on the long-term prognosis of hepatocellular carcinoma patients after liver resection: a systematic review and meta-analysis. Ann Transl Med 2020;8(24):1683. doi: 10.21037/atm-20-4698 with bile duct tumor thrombus: a clinicopathological analysis of factors predictive of recurrence and outcome after surgery. Medicine 2015;94:e364.

38. Shen Y, Li P, Cui K, et al. Neoadjuvant transcatheter arterial chemoembolization for biliary tumor thrombosis: a retrospective study. Int J Technol Assess Health Care 2016;32:212-7.

39. Imura S, Teraoku H, Yoshikawa M, et al. Potential predictive factors for microvascular invasion in hepatocellular carcinoma classified within the Milan criteria. Int J Clin Oncol 2018;23:98-103.

40. Zhang XP, Gao YZ, Chen ZH, et al. An Eastern Hepatobiliary Surgery Hospital/Portal Vein Tumor Thrombus Scoring System as an Aid to Decision Making on Hepatectomy for Hepatocellular Carcinoma Patients With Portal Vein Tumor Thrombus: A Multicenter Study. Hepatology 2019;69:2076-90. 
Table S1 Characteristics of the BDTT group and the classification method of BDTT in each of the included studies

\begin{tabular}{|c|c|c|c|c|c|c|}
\hline \multirow{2}{*}{ References } & \multirow{2}{*}{$\begin{array}{c}\text { No. of } \\
\text { patients }^{\dagger}\end{array}$} & \multicolumn{2}{|c|}{ BDTT type f $^{\ddagger}$} & \multicolumn{2}{|c|}{ Obstructive jaundice } & \multirow{2}{*}{ Classification method of $\mathrm{BDTT}^{\S}$} \\
\hline & & Macroscopic & Microscopic & Presence & Absence & \\
\hline Shiomo et al. & 17 & $17(100 \%)$ & $0(0 \%)$ & $10(58.8 \%)$ & $7(41.2 \%)$ & Not mentioned in the original article. \\
\hline Yeh et al. & 16 & $16(100 \%)$ & $0(0 \%)$ & $16(100 \%)$ & $0(0 \%)$ & Not mentioned in the original article. \\
\hline Ikenaga et al. & 15 & $10(66.7 \%)$ & $5(33.3 \%)$ & $4(26.7 \%)$ & $11(73.3 \%)$ & $\begin{array}{l}\text { Bile duct invasion was classified as b1 (invasion of the third order or more } \\
\text { peripheral branches of the bile duct, but not of second order branches), } \\
\text { b2 (invasion of the second order branches of the bile duct), b3 (invasion } \\
\text { of the first order branches of the bile duct), or b4 (invasion of the common } \\
\text { hepatic duct). Peripheral microscopic biliary invasion was categorized as } \\
\text { b1, and macroscopic biliary invasion was categorized as b2-b4. }\end{array}$ \\
\hline Shao et al. & 27 & $24(88.9 \%)$ & $3(11.1 \%)$ & NA & NA & $\begin{array}{l}\text { Bile duct invasion in the intrahepatic or extrahepatic bile duct was } \\
\text { classified into two categories according to the following criteria: } \\
\text { microscopic bile duct invasion, which represents that the tumor thrombus } \\
\text { can be seen in more than second branch of the biliary tree (excluding } \\
\text { the second order branch), and macroscopic bile duct invasion, which } \\
\text { represents that tumor thrombus was found in no more than the second } \\
\text { order branch, i.e., in the common bile duct, the right or left main hepatic } \\
\text { duct, or the second order branch of the intrahepatic bile duct. }\end{array}$ \\
\hline Yu et al. & 20 & $16(80 \%)$ & $4(20 \%)$ & $14(70 \%)$ & $6(30 \%)$ & $\begin{array}{l}\text { Microscopic BDTT was defined as tumor thrombi limited to the } \\
\text { intrahepatic bile duct, whereas macroscopic BDTT was termed as tumor } \\
\text { thrombi in both the intra- and extra-hepatic bile duct. }\end{array}$ \\
\hline Noda et al. & 22 & $22(100 \%)$ & $0(0 \%)$ & $8(36.4 \%)$ & $14(63.6 \%)$ & Not mentioned in the original article. \\
\hline Meng et al. & 35 & $28(80 \%)$ & $7(20 \%)$ & $17(48.6 \%)$ & $18(51.4 \%)$ & $\begin{array}{l}\text { Bile duct invasion was classified as B1, central type (invasion of } \\
\text { common hepatic duct or first-order branch of bile ducts with or without } \\
\text { microscopic invasion of intrahepatic peripheral bile duct); B2, peripheral } \\
\text { type (invasion of second-order or more peripheral branches of bile duct } \\
\text { but no invasion of first-order branch or common hepatic duct). }\end{array}$ \\
\hline Oba et al. & 13 & $13(100 \%)$ & $0(0 \%)$ & NA & NA & $\begin{array}{l}\text { Macroscopic BDTT was defined as b2-4 (tumor thrombus in the common } \\
\text { hepatic duct or the first to second branches of the bile duct). Microscopic } \\
\text { BDTT was defined as b1 (tumor thrombus in the third order or more } \\
\text { peripheral branches of the bile duct, but not in second order branches). }\end{array}$ \\
\hline Wong et al. & 37 & NA & NA & $31(83.8 \%)$ & $6(16.2 \%)$ & Not mentioned in the original article. \\
\hline Rammohan et al. & 39 & NA & NA & $18(46.2 \%)$ & $21(53.8 \%)$ & $\begin{array}{l}\text { BDTT was classified according to the location of tumor thrombus, as } \\
\text { proposed by Ueda et al. (Type 1: involving the second order intrahepatic } \\
\text { duct; Type 2: involving the first order intrahepatic duct, Type 3a: } \\
\text { extending to the hepatic confluence; Type 3b: implanted tumour growing } \\
\text { in the common hepatic duct (CHD); Type 4: dislodged BDTT within the } \\
\text { CHD). However, the original text did not point to the method about how } \\
\text { to distinguish macroscopic BDTT and microscopic BDTT. }\end{array}$ \\
\hline Kim et al. & 31 & $0(0 \%)$ & $31(100 \%)$ & $0(0 \%)$ & $31(100 \%)$ & $\begin{array}{l}\text { Microscopic BDTT was termed as incidentally discovered tumor thrombi } \\
\text { located in the peripheral bile ducts. Nevertheless, the specific definition } \\
\text { of microscopic BDTT was not made. }\end{array}$ \\
\hline Orimo et al. & 42 & $21(50 \%)$ & $21(50 \%)$ & $6(14.3 \%)$ & $36(85.7 \%)$ & $\begin{array}{l}\text { Microscopic BDTT was defined as BDTT that developed in more than } \\
\text { only the second branch of the intrahepatic bile duct. Macroscopic BDTT } \\
\text { was defined as BDTT existing in the second or first branch of the biliary } \\
\text { tree or the common bile duct. }\end{array}$ \\
\hline Wang et al. & 28 & $12(42.9 \%)$ & $16(57.1 \%)$ & $12(42.9 \%)$ & $16(57.1 \%)$ & $\begin{array}{l}\text { BDTT was divided into two types: microscopic BDTT, which can be } \\
\text { observed beyond the second-order branch of the biliary tree, and } \\
\text { macroscopic BDTT, which is restricted to the second-order branch. }\end{array}$ \\
\hline Pang et al. & 35 & $29(82.9 \%)$ & $6(17.1 \%)$ & NA & NA & Not mentioned in the original article. \\
\hline Yang et al. & 107 & $97(90.7 \%)$ & $10(9.3 \%)$ & $65(60.7 \%)$ & $42(39.3 \%)$ & $\begin{array}{l}\text { Macroscopic BDTT was defined as tumor thrombus in the common } \\
\text { hepatic ducts or the first to second branches of the bile duct. } \\
\text { Microscopic BDTT was defined as tumor thrombus in the third order or } \\
\text { lower peripheral branches of the bile duct. }\end{array}$ \\
\hline
\end{tabular}

BDTT, bile duct tumor thrombus; NA, data not available. ${ }^{\dagger}$, This indicated the number of patients in the BDTT group. ${ }^{\ddagger}$, BDTT was divided into two types macroscopic and microscopic BDTT-in most of the studies. Some of the studies showed the number of patients with macroscopic or microscopic BDTT, although the classification method of BDTT was not mentioned in the text. ${ }^{\S}$, The classification method of BDTT was cited from the original text of the included studies with or without mild modifications. 
Table S2 Survival outcomes of HCC patients with or without BDTT after hepatectomy

\begin{tabular}{|c|c|c|c|c|c|c|c|c|}
\hline References & \multicolumn{4}{|c|}{ BDTT group } & \multicolumn{4}{|c|}{ Non-BDTT group } \\
\hline Yeh et al. & 21.3 & 60.0 & 20.0 & 6.7 & 30.1 & 70.2 & 46.8 & 33.0 \\
\hline Yu et al. & 15.1 & 73.1 & 20.6 & 18.5 & 37.8 & 72.2 & 53.5 & 43.4 \\
\hline Noda et al. & 17.6 & 62.0 & 30.0 & 30.0 & 75.6 & 89.0 & 73.0 & 61.0 \\
\hline Meng et al. & 7.9 & 35.3 & 19.1 & 10.3 & 25.2 & 71.1 & 35.1 & 19.2 \\
\hline Kim et al. & 60.0 & 90.1 & 61.0 & 42.4 & NA & 86.4 & 84.4 & 83.9 \\
\hline Orimo et al. & 29.5 & 75.1 & 44.9 & 36.6 & 91.4 & 89.0 & 72.4 & 61.9 \\
\hline Wang et al. & 31.4 & 81.8 & 50.0 & 37.5 & 68.8 & 90.9 & 66.9 & 55.9 \\
\hline Pang et al. & 15.2 & 68.8 & 19.9 & 16.6 & 80.7 & 84.0 & 64.0 & 55.0 \\
\hline Yang et al. & 16.6 & 60.5 & 20.1 & 12.0 & 84.0 & 84.9 & 69.9 & 57.6 \\
\hline
\end{tabular}

HCC, hepatocellular carcinoma; BDTT, bile duct tumor thrombus; MOST, median overall survival time; OS, overall survival; NA, data not available.

A

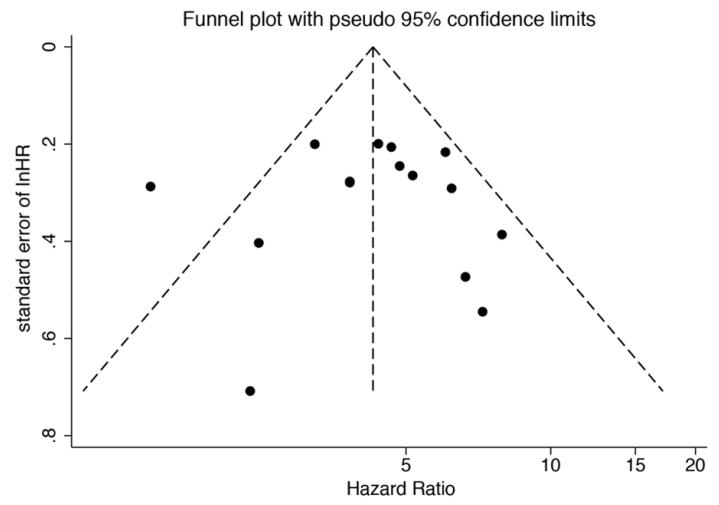

B

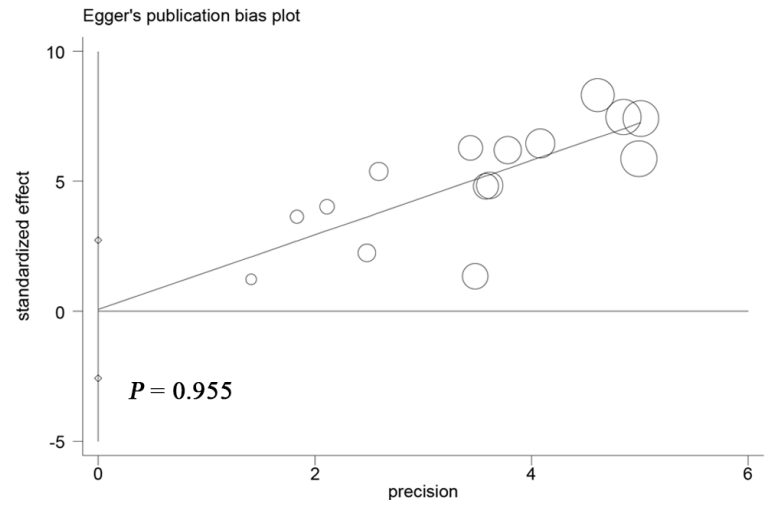

Figure S1 Funnel plot (A) and Egger's plot (B) for the evaluation of the publication bias of HR. 


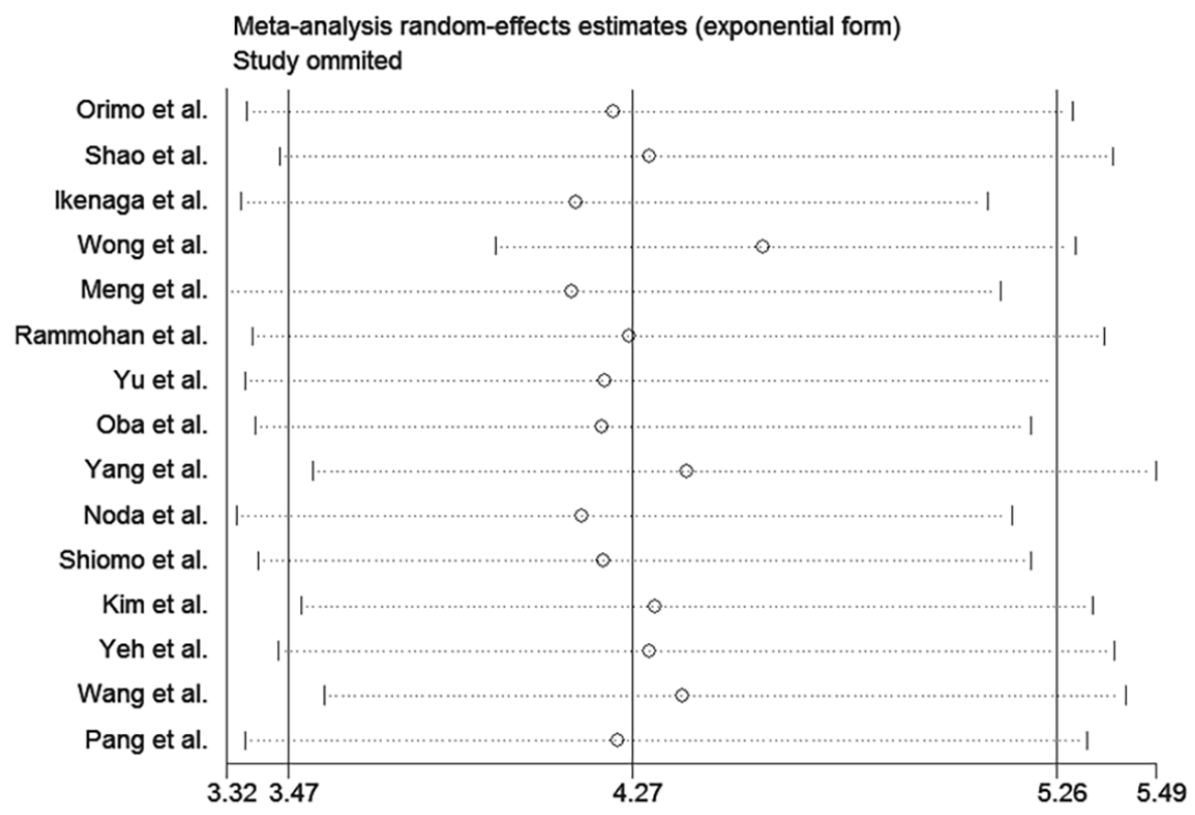

Figure S2 Sensitivity analysis for the evaluation of the potential heterogeneity of HR. 